\title{
Longitudinal Residential Ambient Monitoring: Correlating Sensor Data to Functional Health Status
}

\author{
Saskia Robben \\ Amsterdam University of Applied Sciences \\ P.O. Box 1025, 1000 BA Amsterdam, The Netherlands \\ s.m.b.robben@hva.nl
}

\begin{abstract}
Wireless sensor networks are becoming popular in the field of ambient assisted living. In this paper we report our study on the relationship between a functional health metric and features derived from the sensor data. Sensor systems are installed in the houses of nine people who are also quarterly visited by an occupational therapist for functional health assessments. Different features are extracted and these are correlated with a metric of functional health (the AMPS motor). Though the sample is small, the results indicate that some features are better in describing the functional health in the population, but individual differences should also be taken into account when developing a sensor system for functional health assessment.
\end{abstract}

\section{INTRODUCTION}

The aging population and the development of technology stimulates the growth of e-Health and telemonitoring systems. Systems have been developed that use a wireless network of sensor nodes that register simple events such as opening a door, movement in a room or pressure on a bed. Such ambient monitoring systems can roughly serve two (interrelated) purposes: the first is generating urgent alarms such as sudden inactivity which may indicate a fall. A second purpose is long-term monitoring of behaviour for detecting less urgent deteriorations. For this last purpose many researchers focus on measuring 'activities of daily living' (ADL) [1] and use supervised methods for learning different activity classes [2].

Instead of modeling explicitly the ADL's, some researchers directly try to evaluate health from sensor data. An interesting methodology is using web-based visualization software for evaluating the sensor data prior to events such as falls or hospitalizations [3]. A related study deployed a sensor monitor system which generated alarms, and feedback of the experts who were actually using the systems was used for improving the algorithms underlying the alarms [4]. Another example is a study which defined circadian rhythms based on the average time spent in a room, deviations from these daily patterns could indicate changes in health status [5]. Features typically used in such research are percentage of time in each room (mobility) and number of events for each sensor(-group) per time interval (agitation) [6] [5]. Another approach is adopting a binary representation of features [2] [7], where features describe the occurrence of sensors or combinations of sensor within a certain time frame. A final interesting approach is mining the sequence of events for patterns which can be clustered and used for improving activity recognition and tracking [8].

In our group we focus on models that directly map features derived from the sensor data to a metric of functional health

\author{
Ben Kröse \\ University of Amsterdam \\ P.O. Box 94323, 1090 GH Amsterdam, The Netherlands \\ b.j.a.krose@uva.nl
}

[9]. The chosen metric is the AMPS (Assessment of Motor and Process Skills) [10], a validated metric of functional health and suggested for such use in a review article on intelligent technology for an aging population [11]. The homes are equipped with sensors and the AMPS score is measured every three months by an occupational therapist. We want to develop models that are able to predict the AMPS score from features derived from the sensor data. In this approach first the question arises which are the best features for such a functional health assessment. Because ranking features according to their correlation coefficient with the outcome variable is a useful method in feature selection [12], analysing the features in such a way already can provide insight in subsequent steps that should be taken. In this paper we address the question which features correlates best with the AMPS, and differentiate between identifying the best global features and the best individualized features. By global features we mean features that generalizes over all users, while individualized features are user-specific.

In the next section we describe the AMPS score and the sensor network, and present a selection of features that are derived from the data. Then we describe an experiment in which we use the data to investigate the correlation between features and AMPS and study global features and the best individualized features. The last section concludes with some suggestions for future work.

\section{APPROACH}

This section provides background information on the health and sensor data used for the experiment.

\section{A. Functional Health Data (AMPS)}

The Assessment of Motor and Process Skills (AMPS) [10] instrument differentiates between motor and process skills. The process score contributes to assessing functional health as it is related to cognitive skills, while the motor skills represents a direct physical decline. Both skill sets contribute to the level of which a person can perform activities of daily living. The AMPS is taken by a trained occupational therapist and comprises 12 items of motor skills and 20 items of process skills. These skills are observed in two of 56 standardized daily activities, these two tasks are chosen together with the therapist to avoid choosing the task either too easy or impossible to perform. An example of such an task (activity) can be 'making and serving coffee'. Scores of all the items are linked to a 
continuous scale of ability in motor or process functioning (range from -3 to 4 ). Scores above the cut-off point in motor skills (2.0) or in process skills (1.0) indicate that persons are able to functioning independently in the community. Though normally a specialist will consider both the motor and process part of the AMPS, in this paper we focus on the physical part of the AMPS score, which ranges from -3 to 4 .

\section{B. Sensor Data}

Each participant had a binary ambient sensor network installed in their home which continuously collects data of approximately 16 sensors such as motion sensors (passive infrared), magnetic sensors, and optionally a floating sensor for the toilet and a bed mat for in the bedroom. The behaviour of the residents triggers a sensor in sending an event to a local base-unit where the sensor events are stored in a database. After sending an event a sensor goes to sleep for a short period (10 seconds to 3 minutes depending on the sensor) to save batteries. The participants are recruited from two different assisted living apartment buildings (A and B) where elderly can live independently. The apartment layout in the two buildings is somewhat different, but the sensors are installed in generic locations (e.g. a 'Fridge') which makes the data comparable. Location A was set up first, while the B location followed later, therefore more data is available from the A location than from the B location. All sensor systems have similar technology and design principles as van Kasteren et al. [2].

\section{Features}

For using sensor data as an functional health instrument identifying general patterns (e.g. changes over three months) is more important than detecting outliers. Therefore directly evaluating a single day or single activities is less important. When more days are evaluated simultaneously the sensor data as health instrument is less prone to outliers, a week makes sense as each of the different days is incorporate once. The sensor network generates sensor events which are stored in a database. Each sensor event $e$ is a tuple:

(label,timestamp). For comparison with the AMPS one week of sensor events $(E)$ is considered a data point. This is a high dimensional vector and by means of feature extraction the dimension is reduced. Some of the resulting features are based on sensor counts, others on an inferred location, this is described below.

1) Raw sensor count feature: This feature is based on the number of sensor firings of all the sensors during one week. The set of sensor events is indicated with $E$, and $f_{R}$ is used to denote this feature.

$$
f_{R}=\sum_{e \in E} 1
$$

2) Proportional location features: This feature set is based on the number of firings of a group of sensors, normalized for the total number of features. Again, the set of sensor events with $E$, the set of sensors is indicated with $S$ and $F_{P}$ is used to denote a set of features. For each sensor $s \in S$ a location category $\{$ Bedroom, Bathroom, Kitchen, Livingroom, Doors $\} \in L$ is assigned. Then for each location $l \in L$ a feature is calculated:

$$
f_{P l}=\frac{1}{|E|} \sum_{e \in E}\left\{\begin{array}{l}
1 \text { if } e=l \\
0
\end{array}\right.
$$

3) Location inference features: This set consist of features which represent time spent in a certain location in the house. The apartment of an elderly person consists of several locations where the person can be: 'Bedroom', 'Bathroom', 'Kitchen' and 'Livingroom'. A person can also leave the house (i.e. 'Outside'). Based on the sensor data the location of the person is inferred, and it is possible to track the location of a resident. The algorithm:

1) Select: Extract data point with events $E$ from the database

2) Label: Assign a category $\{$ Bedroom, Bathroom, Kitchen, Livingroom, Outside $\} \in L$ to each sensor event based on sensor location

3) Segment: Replace consecutive series of events with the same label with two instances: an item marking the start and an item marking the end of the series.

4) Label time slots: Determine a label for each minute on each day (by means of the following procedure):

- Use the start and end marking of a series as boundaries. Each time slot in between gets the associated label.

- This results in an matrix sized $n^{*} \#$ days, where $\mathrm{n}=1440$ (the number of minutes in a day) and \#days=7

5) Feature extraction: Calculate the time spent in each location. The set of locations is indicated with $L$, the set of labeled time slots with $T$, and $F_{C}$ is used to denote a set of features. For each location $l \in L$ :

$$
f_{C l}=\sum_{t_{i} \in T}\left\{\begin{array}{l}
1 \text { if } L\left(t_{i}\right)=l_{a} \\
0
\end{array}\right.
$$

The algorithm described above is most accurate in assessing a persons location under the assumption that a person is always somewhere. But as the sensor network sometimes loses track of the person (e.g. due to a small extra room which lack sensors) the algorithm might be assigning a wrong location. However, from a machine learning perspective it is not a problem that the concepts underlying the feature sets might represent something else.

4) Transition feature set: The locations are used to calculate the total number of transitions between locations, which is used as a feature. The set of locations is indicated with $L$, the set of labeled time slots with $T$, and $f_{t}$ is used to denote the transition feature.

$$
f_{t}=\sum_{t_{i} \in T}\left\{\begin{array}{l}
0 \text { if } L\left(t_{i}\right)=L\left(t_{i+1}\right) \\
1
\end{array}\right.
$$

\section{EXPERIMENT}

The goal of this experiment is determining the features that correlates best with functional health in both a group of people and within individuals. 
TABLE I. PARTICIPANT STATISTICS. 'LOCATION' INDICATES IN WHICH APARTMENT COMPLEX THE PARTICIPANT LIVES. 'START' AND 'END' INDICATE THE PERIOD OF PARTICIPATION IN THE SENSOR PROJECT. 'DATA' INDICATES THE NUMBER OF DATA POINTS WHERE BOTH AMPS AND SENSOR DATA IS AVAILABLE.

\begin{tabular}{|l|l|l|l|l|}
\hline Person & Location & Start & End & \#data \\
\hline \hline 1 & A & $2011-06$ & $2012-03$ & 3 \\
\hline 2 & A & $2010-07$ & & 4 \\
\hline 3 & A & $2011-06$ & & 5 \\
\hline 4 & A & $2011-06$ & & 4 \\
\hline 5 & A & $2011-06$ & & 4 \\
\hline 6 & B & $2012-07$ & & 1 \\
\hline 7 & B & $2012-10$ & $2012-12$ & 1 \\
\hline 8 & B & $2012-10$ & & 1 \\
\hline 9 & B & $2012-10$ & & 1 \\
\hline
\end{tabular}

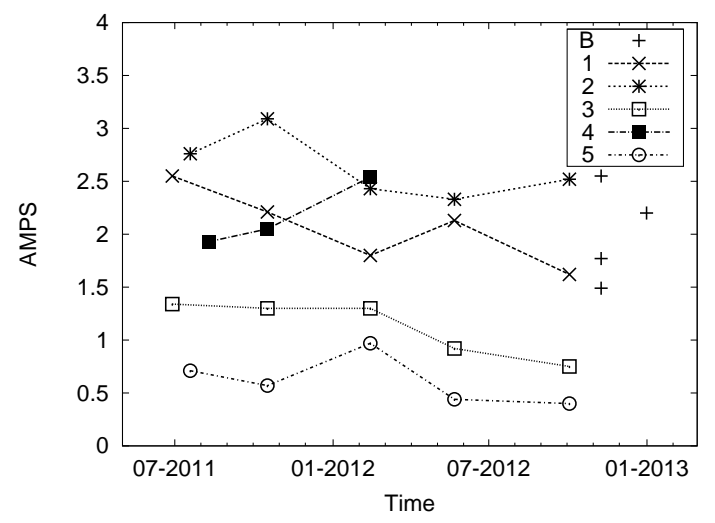

Fig. 1. Original AMPS measurements. Time series of five individuals, B indicates the people of whom only one observation is available.

\section{A. Participants}

Data is collected from 9 subjects in two apartment buildings (A and B). The subjects in location $\mathrm{A}$ have sensors installed since the summer of 2011 (one subject died). The subjects in location B joined in fall of 2012. Some relevant statistics of the subjects are provided in table I. Their scores on functional health (AMPS) are provided in Fig. 1. Some of the subjects also participate in related research activities such as interviews on their attitude towards sensor monitoring [13].

\section{B. Methods}

Sensor and functional status data are selected from nine subjects, resulting in 24 data points. Of five participants more than two data points are available (see table I) and for them also a separate analysis is done. For calculation of the correlation coefficient between the features and the AMPS data Pearson's correlation coefficient is used. The statistical software $\mathrm{R}$ is used for calculating the accompanying confidence intervals, and it is reported whether a result is significant. In the global setup this analysis is done for all of the available data $(\mathrm{N}=24)$, thus the AMPS data of all persons are correlated with features of all persons. In the individual setup this is done separately for each of the five individuals with three or more data points. Because it is more likely to find large effect sizes in small samples, sample size has to be taken into account when interpreting the results. The effect size in a small sample has to be larger to be significant.
TABLE II. RESULTS OF CORRELATION COEFFICIENTS (PEARSON'S R) FOR EACH FEATURE; $*=p<0.1, * *=p<0.05, * * *=p<0.01$

\begin{tabular}{|l|l|l|l|l|l|l|}
\hline & Global & \multicolumn{3}{|l|}{ Individual } & & \\
Feature & All $(\mathrm{n}=24)$ & $1(\mathrm{n}=3)$ & $2(\mathrm{n}=4)$ & $3(\mathrm{n}=5)$ & $4(\mathrm{n}=4)$ & $5(\mathrm{n}=4)$ \\
\hline \hline$f_{R}$ & 0.09 & 0.40 & -0.38 & 0.61 & -0.57 & -0.39 \\
\hline$f_{\text {Cliving }}$ & 0.27 & 0.68 & -0.79 & 0.14 & -0.74 & 0.57 \\
\hline$f_{\text {Cbathroom }}$ & -0.01 & 0.69 & 0.62 & 0.34 & $0.97^{* *}$ & 0.27 \\
\hline$f_{\text {Cbedroom }}$ & -0.03 & 0.34 & -0.11 & -0.07 & -0.07 & $-1.00^{* * *}$ \\
\hline$f_{\text {Coutside }}$ & -0.24 & -0.86 & -0.07 & $0.98^{* * *}$ & 0.13 & -0.27 \\
\hline$f_{\text {Ckitchen }}$ & 0.18 & 0.41 & $0.91^{*}$ & 0.61 & 0.71 & 0.15 \\
\hline$f_{\text {Pliving }}$ & -0.13 & $1.00^{* * *}$ & $-0.98^{* *}$ & 0.12 & $-0.90^{*}$ & 0.51 \\
\hline$f_{\text {Pbathroom }}$ & 0.31 & 0.94 & 0.80 & $-0.87^{*}$ & $0.93^{*}$ & 0.51 \\
\hline$f_{\text {Pbedroom }}$ & $-0.43^{* *}$ & -0.14 & 0.24 & -0.18 & 0.20 & -0.65 \\
\hline$f_{\text {Pdoors }}$ & 0.10 & -0.68 & 0.56 & -0.76 & 0.54 & -0.39 \\
\hline$f_{P \text { itchen }}$ & 0.14 & 0.87 & 0.85 & 0.73 & 0.85 & 0.66 \\
\hline$f_{T}$ & 0.32 & 0.78 & -0.14 & 0.64 & -0.49 & $-0.99^{* *}$ \\
\hline
\end{tabular}

\section{Results and Discussion}

Table II provides the results of the experiment, in the first column the results of the global setup and in the remaining columns the results of the individual setup are given.

1) Global setup: In the global setup only $f_{\text {Pbedroom }}$ yield a significant (negative) correlation with AMPS, this can be interpreted as the more activity in the bedroom (proportional to other activities), the lower is their functional health status. The relation between this feature and the AMPS is visualized in Fig. 2. Additionally there are trends that spending more time in the living room $\left(f_{\text {Cliving }}\right)$ and more activity in the bathroom $\left(f_{\text {Pbathroom }}\right)$ positively correlates with functional health. On the other hand time spent outside ( $f_{\text {Coutside }}$ ) negatively correlates with the AMPS, which means that if people have a low functional health they stay indoors. The last trend worth mentioning is that the transition feature $\left(f_{T}\right)$, which can be regarded as a measure of general activity in the home, positively correlates with functional health (Fig. 3).

2) Individual setup: The other columns in table II provide the correlation coefficients of the features for each of the five individuals. These analyses yield more significant results, but interestingly the direction of the correlation sometimes is opposite for different individuals. For example the proportional activity in the living room $f_{\text {Pliving }}$ significantly correlates for three persons, but in two cases this relation is negative and in the other it is positive. Similar patterns can be seen for other features, for example in Fig. 3 where the transition feature $\left(f_{T}\right)$ is visualised. While in the global setup there was a trend that this feature positively correlates with the AMPS (the more active the more healthier in general). Though for two individuals this feature negatively correlates with the AMPS, which can be interpreted as restlessness in the behaviour of these individuals indicate that something is wrong.

A final interesting result are the individual differences on the two features related to leaving and entering the house. For person 3 the feature $f_{\text {Coutside }}$ positively correlates with the AMPS, meaning that the she stays inside as her functional status is low. Correspondingly the negative correlation of the feature $f_{P \text { doors }}$ indicate that the door is used more when she functions bad. This can indicate that she leaves the house more frequently but for shorter periods of time or that she receives more visitors (either care professionals or family). Although this makes sense, this pattern is not seen in all the individuals, where sometimes the $f_{P d o o r s}$ positively correlates with the functional status. This can be explained by the fact 


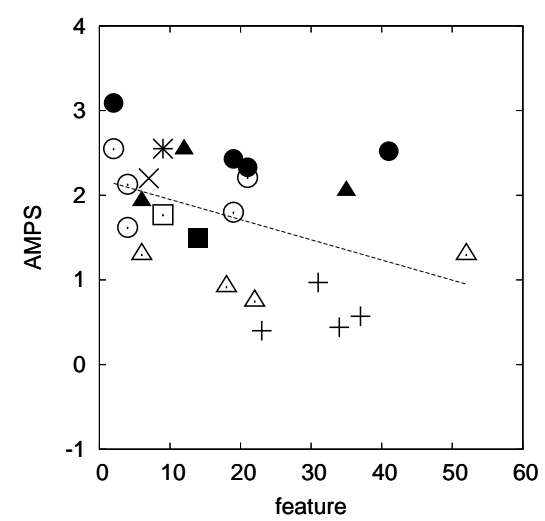

Fig. 2. $f_{\text {Pbedroom: }}$ : Feature representing activity in bedroom. In the global setup this feature negatively correlates with the AMPS. Different icons refer to different individuals.

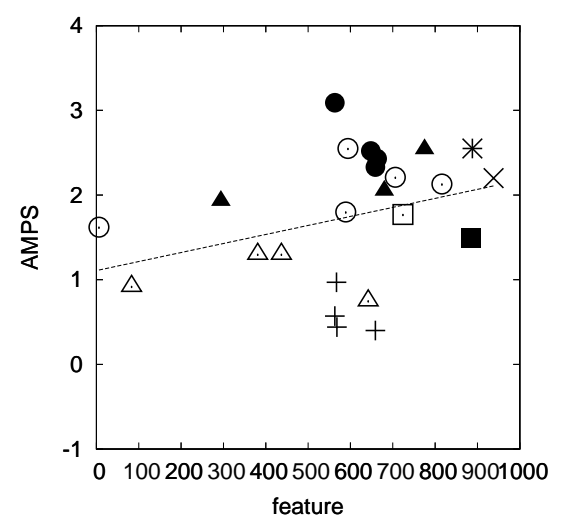

Fig. 3. $f_{T}$ : Feature representing transitions between locations. Different icons refer to different individuals.

that when people have a low functional status they tend to isolate themselves.

\section{CONCLUSIONS AND Future WORK}

The results indicate that for assessing the functional health status of a person only a few features from the sensor data generalize over the population. However, in developing a sensor system as a functional health assessment tool it should be taken into account that the behaviour between people varies enormously. As the results showed, individualized analysis yielded features that positively correlate with the functional health status for some persons and negatively for others. Change seems important for these features, and therefore this can be used as an argument for using a baseline measurement for comparing the changes. It should be noted again that in this study data from only a limited number of participants was available.

One part of the future work should be focused on obtaining better global features, because this can contribute to a system for functional health assessment. The input of health care specialists can be of great value here, as their current assessment instruments and experience can inspire the feature extraction process. On the other hand individual differences should be examined carefully, as our experiment showed that these can have predictive value. Recruiting volunteers to participate for a longer period of time can be quite an undertaking, and this was also reflected in the low number of data in this study. However, there are currently more research groups doing field studies with ambient monitoring systems. If functional health status of all the participants in these studies is assessed with similar instruments, the research community can work together. More sensor and health data combined can boost the development of an automated functional health assessment system.

\section{ACKNOWLEDGMENTS}

The authors would like to thank Margriet Pol, Tiska Ikking and the elderly participants. This work is supported by the Agentschap NL projects Health-lab and Design for Wellbeing and SIA project Smart Systems for Smart Services.

\section{REFERENCES}

[1] S. Katz, A. Ford, R. Moskowitz, B. Jackson, and M. Jaffe, "Studies of illness in the aged," JAMA: the journal of the American Medical Association, vol. 185, no. 12, pp. 914-919, 1963.

[2] T. van Kasteren, A. Noulas, G. Englebienne, and B. Kröse, "Accurate activity recognition in a home setting," in Proceedings of the 10th international conference on Ubiquitous computing. ACM, 2008, pp. $1-9$.

[3] M. Rantz, M. Skubic, and S. Miller, "Using sensor technology to augment traditional healthcare," in Engineering in Medicine and Biology Society, 2009. EMBC 2009. Annual International Conference of the IEEE. IEEE, 2009, pp. 6159-6162.

[4] M. J. Rantz, M. Skubic, R. J. Koopman, G. L. Alexander, L. Phillips, K. Musterman, J. Back, M. A. Aud, C. Galambos, R. D. Guevara et al., "Automated technology to speed recognition of signs of illness in older adults," Journal of Gerontological Nursing, vol. 38, no. 4, p. 18, 2012.

[5] G. Virone, M. Alwan, S. Dalal, S. Kell, B. Turner, J. Stankovic, and R. Felder, "Behavioral patterns of older adults in assisted living," Information Technology in Biomedicine, IEEE Transactions on, vol. 12, no. 3, pp. 387-398, 2008.

[6] A. Fleury, M. Vacher, and N. Noury, "Svm-based multimodal classification of activities of daily living in health smart homes: sensors, algorithms, and first experimental results," Information Technology in Biomedicine, IEEE Transactions on, vol. 14, no. 2, pp. 274-283, 2010.

[7] E. Tapia, S. Intille, and K. Larson, "Activity recognition in the home using simple and ubiquitous sensors," Pervasive Computing, pp. 158175, 2004.

[8] P. Rashidi, D. J. Cook, L. B. Holder, and M. Schmitter-Edgecombe, "Discovering activities to recognize and track in a smart environment," Knowledge and Data Engineering, IEEE Transactions on, vol. 23, no. 4, pp. 527-539, 2011.

[9] S. Robben, G. Englebienne, M. Pol, and B. Kröse, "How is grandma doing? predicting functional health status from binary ambient sensor data," in 2012 AAAI Fall Symposium Series, 2012, pp. 26-31.

[10] A. Fisher, Assessment of motor and process skills. Three Star Press Fort Collins, CO, 1999.

[11] M. Pollack, "Intelligent technology for an aging population: The use of ai to assist elders with cognitive impairment," AI magazine, vol. 26, no. 2, p. 9, 2005.

[12] M. A. Hall, "Correlation-based feature selection for machine learning," Ph.D. dissertation, The University of Waikato, 1999.

[13] M. Kanis, S. Alizadeh, J. Groen, M. Khalili, S. Robben, S. Bakkes, and B. Kröse, "Ambient monitoring from an elderly-centred design perspective: What, who and how," Ambient Intelligence, pp. 330-334, 2011. 